\title{
Adoption des Technologies Agro-Écologiques : Perception et Préférence des Agriculteurs de la Zone Cotonnière du Bénin
}

\author{
Léon H. Akpatcho, \\ Unité de Coordination du Projet d'appui à la Transition Agro-écologique \\ dans les Zones Cotonnières du Bénin, Institut National des Recherches \\ Agricoles du Bénin \\ Gerard C. Zoundji, \\ Université Nationale d'Agriculture de Porto-Novo, Bénin
}

Fifanou G. Vodouhê,

Laboratoire d'Analyses et de Recherches sur les Dynamiques Économiques et Sociales Faculté d'Agronomie, Université de Parakou, Bénin

Doi:10.19044/esj.2019.v15n30p146 URL:http://dx.doi.org/10.19044/esj.2019.v15n30p146

\section{Résumé}

La promotion des pratiques agro-écologiques est devenue l'une des options favorables pouvant contribuer durablement à la gestion des sols et à la préservation des écosystèmes pour la sécurité alimentaire et nutritionnelle dans le contexte actuel des changements climatiques. Cette étude vise à analyser la perception et la préférence des producteurs sur les pratiques agroécologiques innovantes promues dans le cadre du «projet d'appui à la Transition Agro-écologique dans les Zones Cotonnières du Bénin (TAZCO) ». Pour faciliter l'adoption de telles technologies par les producteurs, il faut agir sur les déterminants qui leurs sont favorables. La perception étant un déterminant de l'adoption et la présente étude a été réalisée pour proposer par commune, au moins cinq technologies pour lesquelles les producteurs ont une bonne préférence. Cette étude a été conduite dans la zone d'intervention du TAZCO, constituée de Banikoara, Kandi, Oussa-Péhunco, Parakou et Savalou. En utilisant la méthode d'échantillonnage aléatoire avec le paramètre de disponibilité des producteurs impliqués, 282 producteurs ont été enquêtés lors de la collecte des données parmi les 300 impliqués dans la phase pilote du projet. Les données ont été collectées à l'aide d'un questionnaire structuré, développé sur smartphones avec l'application ''KoBocollect', Ces données ont été analysées à l'aide de méthode multicritère d'aide à la décision et une liste de cinq technologies variant d'une zone à l'autre a été établie. L'étude a montré que les technologies de la famille «Gestion Intégrée de la Fertilité du 
Sol» et «Gestion Intégrée Agriculture-Elevage »viennent en tête dans la majorité des zones d'intervention du TAZCO.

Mots clés : Agriculture durable, Pratique agro-écologique, Fertilité des sols, coton, Bénin, WOCAT

\title{
Adoption of Agro-ecological Technologies: Perception and Farmers' Preference in Cotton Production Area in Benin
}

\section{Léon H. Akpatcho,}

Unité de Coordination du Projet d'appui à la Transition Agro-écologique dans les Zones Cotonnières du Bénin, Institut National des Recherches

Agricoles du Bénin

Gérard C. Zoundji,

Université Nationale d'Agriculture de Porto-Novo, Bénin

Fifanou G. Vodouhê,

Laboratoire d'Analyses et de Recherches sur les Dynamiques Économiques et Sociales Faculté d'Agronomie, Université de Parakou, Bénin

\begin{abstract}
Agro-ecological practices promotion become one of the favorable options that can sustainably contribute to soil management and ecosystem preservation for food and nutrition security under climate change context. This study aims to analyze producers' perception and preference on agro-ecological practices promoted by TAZCO project (Agro-ecological Transition in Benin Cotton Areas). To facilitate promoted technologies adoption by producers, it is necessary to act on determinants affecting them positively. Based on the fact that perception is one of the technology adoption determinants, the study was carried out to suggesting each of the municipalities involved in the study at least five technologies which received producers' favorable preference. The study was conducted in the area covered by TAZCO activities (Banikoara, Kandi, Oussa-Péhunco, Parakou and Savalou). Using the random sampling method with producers involved's availability parameter, 282 producers over to the 300 involved in the pilot project were interwied during data collecting. Data were collected using a structured questionnaire developed under "Kobocollect" application on smartphones. The multi-criteria decision-support
\end{abstract}


method was used to analyze collected data and a list of five technologies varying from one zone to another was established. The study showed that the technologies named "Integrated Management of Soil Fertility" and "Integrated Management Agriculture-Livestock" revealed to be the most important in the majority of TAZCO intervention areas.

Keywords: Sustainable agriculture, Agro-ecological practices, Soil fertility, Cotton, Benin, WOCAT

\section{Introduction}

L'agriculture se trouve confrontée à plusieurs contraintes dont l'une des plus importantes est le phénomène de la dégradation des sols qui limite le potentiel de l'agriculture dans la plupart des pays en voie de développement (Serme et $a l ., 2015$ ). Au Bénin, près de $60 \%$ de la surface agricole utile est menacée par une dégradation due à l'assujettissement des terres à de mauvaises pratiques culturales dont la culture itinérante sur brûlis qui détruit la flore, la matière organique ainsi que la faune et la microfaune du sol (Igué et $a l ., 2013)$. Elle s'exprime sous diverses formes : l'érosion hydrique et éolienne, la baisse de fertilité, etc. Il s'en suit une baisse de fertilité dommageable pour la production agricole (MAEP, 2017). Alohou et Agossou (1998) identifient la baisse de fertilité comme l'un des problèmes majeurs qui affectent l'agriculture dans le nord Bénin. Elle a pour causes principales, la forte poussée démographique, doublée d'une extension rapide de la culture cotonnière dans le Nord Bénin (Baco et al., 2002). Cette baisse de la fertilité des sols compromet la durabilité des systèmes de production et demeure un problème majeur pour l'agriculture béninoise, du fait des menaces tant sur la sécurité alimentaire que sur les revenus des producteurs et des effets négatifs sur la réduction de la pauvreté rurale (Adégbola et Adékambi, 2006).

Pour corriger le phénomène, l'amendement chimique a été identifié comme une solution (Akpinfa et al., 2017). Cependant, les technologies de fumure chimique mises au point depuis de longues années par la recherche et diffusées selon la démarche classique de vulgarisation (information, démonstration, application) avec l'appui d'un réseau de magasins pour la distribution d'engrais et un système de crédit pour le financement n'ont eu pour effet que de faire connaître les engrais et leurs efficacités, et laissaient de côté le problème de maintien du taux de matière organique (Adégbola et $a l$., 2016). Cette méthode a montré ses limites et ne peut permettre à elle seule d'arriver à bout des problèmes de baisse de fertilité.

Pour répondre à ces enjeux, il est nécessaire d'opérer une transformation profonde des modes de production et s'orienter vers une approche durable de production telle que l'agro écologie qui cherche à maintenir ou à restaurer la fertilité des sols (Berton et al., 2013). En effet, 
l'agro écologie est un mode de production utilisant au mieux les fonctions des écosystèmes et les processus écologiques pour une agriculture productive et moins nocive pour l'environnement et la santé humaine (Leterme et Morvan, 2010). Elle optimise l'utilisation des ressources locales tout en réduisant au minimum les impacts environnementaux et socio-économiques négatifs des technologies modernes et apporte une meilleure contribution à la sécurité alimentaire et nutritionnelle (FAO, 2014). Les systèmes agricoles dominants n'étant aujourd'hui plus soutenables, l'agro écologie apporte donc des solutions pour produire durablement (Blaimont, 2013).

C'est dans la perspective d'appuyer les pratiques agro écologiques que le «projet d'appui à la Transition Agro-écologique dans les Zones Cotonnières du Bénin (TAZCO) » a été initié par le Gouvernement du Bénin et financé par l'Agence Française du Développement (AFD). Il renforce le développement des pratiques agro écologiques innovantes qui optimisent la mobilisation des processus écologiques dans le domaine de la production agricole et de la sécurité alimentaire et nutritionnelle dans la zone cotonnière du Bénin. Il vise l'amélioration des systèmes de cultures à base de coton par l'introduction de pratiques de la grille WOCAT (World Overview of Conservation Approaches and Technologies) reconnues comme des technologies agro-écologiques (FAO, 2011) dont les effets bénéfiques peuvent induire une augmentation des rendements des cultures pouvant atteindre 170\% (Savadogo et al., 2011). Ces pratiques combinent entre autres cinq familles de technologies agro écologiques : la gestion intégrée de la fertilité des sols (GIFS), la gestion intégrée de l'agriculture et de l'élevage (GIAE), la gestion des eaux du sol, l'agriculture de conservation et l'agroforesterie. Ces pratiques ne sont plus nouvelles au Bénin car des projets antérieurs au TAZCO tels que le Projet « Réhabilitation et protection des sols dégradés et renforcement des instances foncières locales dans les zones rurales » et autres, les ont déjà diffusées dans des zones de production (Kandi, Parakou, etc.). Il convient alors d'agir sur les déterminants favorables à leur adoption en vue de leur mise à échelle.

Parmi les déterminants les plus reconnus pour l'adoption en matière des innovations agricoles, la perception a une place de choix (Cheikho, 2015 ; Vodouhê, 2010). Les risques potentiels liés aux innovations fondent la perception des producteurs; ce qui conduit à l'adoption ou non de l'innovation. Ce point de vue a été confirmé à travers les travaux de Shapiro et al. (1992) qui soutiennent que la perception du risque est un facteur plus déterminant que l'attitude réelle face au risque. À cet effet, Marra et al. (2003), distinguent différents éléments de risque dans le processus de décision comme l'apprentissage (learning) et la perception des producteurs de la distribution des probabilités présentes et futures de profits et de pertes attendus de la technologie, la covariance des profits entre l'ancienne et la nouvelle 
technologie et les délais de retour sur investissement de la technologie. Ainsi, la perception du risque par les producteurs est sous l'influence de facteurs variés qu'il urge de prendre en considération pour garantir l'adoption d'une technologie. C'est pour cette raison que la présente étude s'est donnée comme objectif d'analyser la perception des producteurs, des technologies vulgarisées par le projet TAZCO, afin de ressortir les technologies pour lesquelles les producteurs ont plus de préférence. L'identification de ces technologies permettra de territorialiser les technologies agro écologiques à vulgariser et les mesures d'accompagnement de l'adoption.

\section{Méthodologie}

Les études de référence sur l'analyse de la préférence, adoptent la méthode des profils complets. Chaque répondant est exposé à un ensemble complet de combinaisons d'attributs. Le répondant doit classer les profils ou leur attribuer une note en fonction de ses préférences. La principale limite de cette méthode tient au fait que le nombre d'attributs constituant le profil ne doit pas être trop élevé pour éviter que la tâche cognitive ne soit trop complexe pour le répondant (Guillot-Soulez et Soulez, 2011). Or dans le cadre de cette étude, la préférence des expérimentateurs parmi dix-sept technologies suivant quatre critères a été analysée. Donner ses préférences dans ce cas revient pour l'enquêté à se prononcer en comparant implicitement 17 fois 4 éléments. Ce qui n'est pas facile à faire pour le mental humain. La présente étude s'est donc basée sur une démarche d'analyse simple et rationnelle qui combine l'usage du Front de Pareto, de fonction de désirabilité et du critère unique de synthèse mise au point par Benki (2014). Cette méthode est adoptée pour déterminer la préférence des agriculteurs expérimentateurs pilotes des technologies agroécologiques dans cinq communes du Bénin.

Le Front de Pareto permet de faire un tri selon la domination ou non des alternatives. Une alternative est dite non dominée lorsqu'elle est meilleure à toutes les autres de la série sur au moins un critère et retenue ; dans le cas contraire elle est dominée et laissée de côté. Dans nos séries, les alternatives non dominées étaient une ou deux par communes. Or, il est souhaité que le projet ait au moins cinq technologies alternatives à proposer par commune aux producteurs afin de satisfaire leur besoin de durabilité de la production. D'où la nécessité de repêcher les moins dominées. Pour ce faire, on reconsidère l'ensemble des alternatives retenues après le premier tri (non dominés et moins dominés) et on relativise les scores recueillis par critère en utilisant les fonctions de désirabilité qui permettent de minimiser ceux à minimiser et en maximisant ceux à maximiser selon leur désirabilité. Le critère unique qui agrège les valeurs normées de ces critères permet d'ordonner les alternatives retenues selon les niveaux de préférence qui s'en dégagent. Les tableaux 4 à 18 en annexe résument cette démarche. 


\section{Zones d'étude}

La zone d'investigation de cette étude est constituée des localités dans lesquelles le projet d'appui à la Transition Agro-écologique dans les Zones Cotonnières (TAZCO) met en œuvre ses activités de la phase pilote au Bénin depuis 2017. Il s'agit des cinq communes réparties sur deux pôles de développement agricoles du Bénin : le pôle 2 (Banikoara, Kandi, OuassaPéhunco) et le pôle 4 (Parakou et Savalou). D'une superficie totale de 36782 $\mathrm{km}^{2}$ le Pôle de Développement Agricole 2 représente 32,05 \% du territoire national et se situe entre $10^{\circ} 13^{\prime}$ et $11^{\circ} 18^{\prime}$ de latitude Nord et entre $1^{\circ} 41^{\prime}$ et $3^{\circ} 41^{\prime}$ de longitude Est. Ce pôle qui est le principal bassin cotonnier du Bénin, s'étend sur les 10 communes que sont Banikoara, Kandi, Gogounou, Ségbana, Kèrou, Ouassa-Péhunco, Kouandé, Bembèrèkè, Kalalé et Sinendé. Mais en plus du coton qui est sa culture locomotive, le maïs et le sorgho y sont aussi développés pour exploiter les arrières effets de l'utilisation de l'engrais sur la culture du coton. C'est également la grande zone des cheptels de ruminants conduits en élevage extensif (Plan de développement du pôle agricole 2/MAEP, 2017). La moyenne pluviométrique annuelle des dix dernières années dans cette zone est comprise entre $1000 \mathrm{~mm}$ et $1200 \mathrm{~mm}$. Les températures minimale et maximale y sont respectivement de $21^{\circ} \mathrm{C}$ et de $34,3^{\circ} \mathrm{C}$ avec une moyenne décennale de $27,7^{\circ} \mathrm{C}$ (Station synoptique de Kandi, 2018).

Le pôle de développement agricole 4 constitue une zone de diversification coton-vivrier-anacardier et couvre les communes de Parakou, Nikki, Pèrèrè, N'Dali, Tchaourou, Ouèssè, Savè, Savalou, Bantè, Glazoué, Dassa-Zoumè, Djidja, Bassila, Djougou, Copargo et Ouaké. Il abrite un système d'intégration agriculture, élevage et foresterie basé sur l'anacardier et le coton, comme cultures locomotives. Il y est également développé, le maïs, le sorgho, les racines et tubercules, des légumineuses, le manguier et l'élevage en système extensif (Plan de développement du pôle agricole 4/MAEP, 2017). C'est une zone à forte potentialité agricole avec une faible densité d'occupation des terroirs en dehors de Parakou (Nougbodohoué, 2017). Ces conditions permettent de cultiver une grande diversité de culture (Sinsin et al., 2013). A Parakou, le climat est de type soudano-guinéen, soit tropical humide, avec une saison de pluies de mai à octobre et une saison sèche de novembre à avril. La moyenne annuelle des températures est de $26,6^{\circ} \mathrm{C}$. La précipitation moyenne annuelle est de $1200 \mathrm{~mm}$ avec un pic entre juillet et septembre (CARDER, 2013). Tandis que Savalou est une zone agro-écologique soudanoguinéenne de transition comprise entre $7^{\circ} 35^{\prime}$ et $8^{\circ} 13^{\prime}$ latitude-Nord d'une part et entre $1^{\circ} 3^{\prime}$ et $2^{\circ} 6^{\prime}$ longitude-Est d'autre part. Son relief culmine entre 120 et $500 \mathrm{~m}$ avec des pentes qui varient entre 3 et $10 \%$ dans les sites agglomérés et elle est caractérisée par un climat bimodal à deux saisons des pluies avec une moyenne pluviométrique annuelle comprise entre1 000 et $1200 \mathrm{~mm}$. Les sols 
sont de type ferrugineux tropical lessivés. Elle est une plaine érodée développée sur du gneiss et laissant en relief des éléments granitiques ou ferrugineux résistants (Guerin, 2017).

\section{Unités de recherche}

L'échantillon d'étude a porté sur les producteurs expérimentateurs du projet TAZCO de chacun des dix villages pilotes répartis dans les cinq Communes. Le nombre de producteurs enquêtés par Commune est fonction de leur disponibilité à participer à la collecte des données. Ainsi, 282 producteurs ont été enquêtés sur un total de 300 producteurs expérimentateurs (Tableau 1).

Tableau 1 : Répartition des enquêtés par genre et par commune

Effectifs des enquêtés

\begin{tabular}{c|c|c|c}
\hline Communes & Hommes & Femmes & Total \\
\hline Banikoara & 59 & 4 & 63 \\
Kandi & 52 & 3 & 55 \\
Ouassa-Péhunco & 50 & 10 & 60 \\
Parakou & 47 & 2 & 49 \\
Savalou & 32 & 23 & 55 \\
\hline Total & 240 & 42 & 282 \\
\hline
\end{tabular}

\section{Collecte et analyse des données}

Les données collectées sont relatives aux activités du projet dans ces communes. Ces activités ont lieu dans dix villages à raison de deux par commune et sont relatives à l'expérimentation et à la démonstration de technologies résilientes aux changements climatiques telles que retenues dans la grille WOCAT. Les différentes technologies agro-écologiques de la grille WOCAT sont : 
Tableau 2 : Grille des technologies WOCAT

\begin{tabular}{|c|c|c|c|c|}
\hline $\begin{array}{l}\text { 1- Gestion Intégrée } \\
\text { de l'Agriculture et de } \\
\text { l'Elevage (GI=AE) }\end{array}$ & $\begin{array}{l}\text { 2- Gestion Intégrée de } \\
\text { la Fertilité des Sols }\end{array}$ & $\begin{array}{l}\text { 3- Conservation } \\
\text { des eaux et des } \\
\text { sols }\end{array}$ & $\begin{array}{l}\text { 4- Agriculture } \\
\text { de conservation }\end{array}$ & 5- Agroforesterie \\
\hline $\begin{array}{c}\text { 1- Production } \\
\text { fourragère herbacée } \\
\text { et arbustive; } \\
\text { 2- Parcage rotatif; } \\
\text { 3- Etable fumière } \\
\text { 4- Haie vive, } \\
\text { embocagement et } \\
\text { diversification }\end{array}$ & $\begin{array}{l}\text { 5- Rotation et } \\
\text { assolement; } \\
\text { 6- Intégration de } \\
\text { légumineuses } \\
\text { amélioratrices; } \\
\text { 7- Jachère améliorée de } \\
\text { cycle court; } \\
\text { 8- Compostage; } \\
\text { 9- BRF (Bois Raméal } \\
\text { Fragmenté) et broyage } \\
\text { des résidus de coton } \\
\text { 10- Intégration de } \\
\text { légumineuses à grain; }\end{array}$ & $\begin{array}{l}\text { 11- Travail } \\
\text { minimum et } \\
\text { localisé } \\
\text { 12- Zaï en ligne } \\
\text { mécanisé }\end{array}$ & $\begin{array}{l}\text { 13- Reprises de } \\
\text { jachère/ } \\
\text { intercultures } \\
\text { améliorées; } \\
\text { 14- Strip-tillage } \\
\text { et Reprise de } \\
\text { zone de } \\
\text { pâturage }\end{array}$ & $\begin{array}{l}\text { 15- Igname sous } \\
\text { couvert ligneux } \\
\text { de Gliricidia; } \\
\text { 16- Culture } \\
\text { annuelle en } \\
\text { intercalaire } \\
\text { d'Anacardier } \\
\text { 17- Arbre dans } \\
\text { paysage } \\
\text { Faidherbia albida }\end{array}$ \\
\hline
\end{tabular}

Source : www.WOCAT.org

Le questionnaire utilisé pour la collecte des données a été développé dans l'application 'KoBocollect' ce qui a permis d'utiliser des smartphones pour la collecte. Ce questionnaire a été développé dans KoBo en raison de son libre et gratuit accès sur internet d'une part, et d'autre part en raison de la familiarité des enquêteurs du projet à son usage. Comme l'a dit Sherin et al. (2018), KoBoToolbox est une entrée de données en ligne gratuite et open source outil développé par l'initiative humanitaire de Harvard avec le soutien de diverses organisations comme Brigham et Women's Hospital, USAID. C'est un outil bien apprécié pour les avantages pratiques qu'il présente. Deniau C. et al. (2017), pensent que l'utilisation de cet outil d'interface numérique présente plusieurs avantages : rapidité d'accès aux données collectées et leur partage, normalisation des données qualitatives pour l'analyse statistique et la pré-analyse effectuée sur la plate-forme de stockage de KoBoToolbox, compilation et création de base de données, accès à distance et partage de données et enfin partage de solutions par la "communauté KoBoToolbox".

Les questions posées aux expérimentateurs sont relatives à leur connaissance des technologies, leur décision de pratiquer ou non ces technologies, leur expérimentation de l'efficacité et du coût des technologies utilisées, leur appréciation des risques liés à chaque technologie expérimentée ainsi que le temps nécessaire à sa réalisation. Les questions étant de type fermées, des choix de réponse étaient proposés aux enquêtés (oui, non, ne sait pas, long, moyen, court, élevées, moyen, bas, difficile, très difficile, acceptable, pas du tout difficile, etc). 
Les données collectées ont été soumises à des analyses quantitatives et qualitatives. L'analyse quantitative concerne la connaissance de chacune des technologies vulgarisées aux fins de comparer la popularité de ces technologies les unes par rapport aux autres. Il s'agit de compter le nombre de connaisseurs et de les comparer par technologies. Ceci parce que certaines appréciations sont plus objectives sur les technologies agricoles lorsqu'on les connait et qu'on les pratique.

En ce qui concerne l'analyse qualitative, elle a concerné l'appréciation des expérimentateurs suivant les critères que sont : la complexité de mise en œuvre de la technologie, le coût opérationnel lié à la mise en œuvre de la technologie, l'efficacité (temps d'effet) de la technologie et le niveau de risque associé à chaque technologie. Il en est ainsi parce que selon la littérature le risque a un poids dans le processus d'adoption. Menapace et al. (2013) ont montré que plus un individu est averse, plus il perçoit de manière importante les risques de pertes. En ce qui concerne l'efficacité, le coût opérationnel et la complexité, Adesina et Zinnah (1993) ont montré que l'avantage relatif perçu par l'agriculteur (efficacité, profitabilité, adaptabilité, etc.) détermine son intérêt pour l'innovation qui lui est apporté. Les méthodes d'analyse utilisées sont dites méthodes d'aide à la décision multicritère (MADM) (Cote et al., 2017). Pour l'utilisation de ces méthodes, il a été d'abord converti les appréciations des expérimentateurs en des valeurs absolues afin de comparer leur fréquence d'apparition en utilisant le logiciel Excel. Pour ce fait, chaque technologie est considérée comme une alternative et les réponses recueillies des bénéficiaires ont permis de distribuer des scores. Le principe de la distribution des scores est que les réponses qui ne permettent pas d'avoir une appréciation objective ont une valeur nulle (non, ne sait pas, vide). Pour les réponses objectives, on donne des scores de « $1 »$ à $« n »$ dans une série de la pire à la meilleure situation espérée. Les valeurs analysées proviennent de la somme des scores recueillis par alternative dans chaque commune.

Trois étapes ont été adoptées dans l'analyse des données:

- L'usage du Front de Pareto pour le premier tri ; cette méthode a été utilisée par (Benki, 2014) qui s'en est servi en conception mécanique multicritère. Le front de Pareto est l'ensemble des alternatives non dominées (alternatives dites Pareto-optimales). Il consiste à comparer les alternatives entre elles selon les critères retenus et à éliminer celles qui sont dominées. Il y a donc autant de comparaison à faire qu'il y a de critères et d'alternatives. Cette méthode est d'utilisation facile lors que les nombres de critères et d'alternatives sont faibles. Mais elle peut être généralisée à un plus grand nombre de critères avec une programmation appropriée de l'outil informatique. Dans le cadre de cette étude, la version de Excel déjà programmée et disponible sur www.1000minds.com/go/supagro a été utilisée 
- L'utilisation de la fonction de désirabilité qui est une normalisation des valeurs obtenues par critère. Il s'agit d'attribuer une valeur comprise entre 0 et 1 ou $0 \%$ et $100 \%$ aux différentes alternatives par critère à l'aide d'une fonction exprimant un degré de satisfaction en fonction de ces valeurs. Elle peut se faire de différentes manières.

Exemple :

$\bigcirc$ pour un critère à maximiser : $C N_{i j}=\frac{C_{i j}}{\operatorname{maxi}\left(C_{i j}\right)}$

$\circ$ pour un critère à minimiser : $C N_{i j}=\frac{\min \left(c_{i j}\right)}{C_{i j}}$

○ Autres possibilités : $C N_{i j}=\frac{C_{i j}}{\sqrt{\sum C_{i j}^{2}}}$

Avec :

- $\mathrm{CN}_{\mathrm{ij}}$ : valeur normée d'un critère donné ;

- $\mathrm{C}_{\mathrm{ij}}$ : valeur réelle quelconque d'un critère donné dans la série ;

- $\max _{\mathrm{i}}\left(\mathrm{C}_{\mathrm{ij}}\right)$ : plus grande valeur observée d'un critère donné dans la série ;

- $\min _{\mathrm{i}}\left(\mathrm{C}_{\mathrm{ij}}\right)$ : plus petite valeur observée d'un critère donné dans la série ;

- $\mathrm{ij}$ : position de la valeur dans la matrice.

- la détermination d'une valeur unique pour comparer les alternatives. Pour ce faire, il faut donner un poids au critère d'appréciation à utiliser dans les calculs. Ce qui mous a amené à demander aux producteurs de dire l'importance des critères qui les déterminent à adopter ou non une technologie agroécologique en donnant une note à chaque critère sur une grille de 1 à 4 . Le moins important : 1 , le suivant 2 , le troisième 3 et le meilleur 4. La somme des notes attribuées par un producteur est alors de 10 (soit $1+2+3+4=10$ ). Cette méthode permet d'établir une sorte de consensus sur le poids de chaque critère selon la perception des expérimentateurs qui ont dans ce cas un avis d'expert. Elle s'apparente donc à la méthode dite Delphi, utilisée par Ekionea (2011) pour obtenir un consensus sur les capacités à développer pour une bonne gestion des connaissances. Elle permet de consigner les opinions d'experts dans un domaine précis. En effet, la méthode Delphi a pour but de rassembler des avis d'experts sur un sujet précis et de mettre en évidence des convergences et des consensus sur un sujet en soumettant ces experts à des vagues successives de questionnements. Cette méthode trouve toute son utilité là où de nombreuses incertitudes planent sur la définition précise d'un sujet et où de nombreuses questions sont restées sans réponses satisfaisantes. 


\section{Résultats}

\section{Poids des critères d'appréciation selon les producteurs}

Les notes attribuées par les producteurs enquêtés aux différents critères ont permis de construire la figure ci-après :

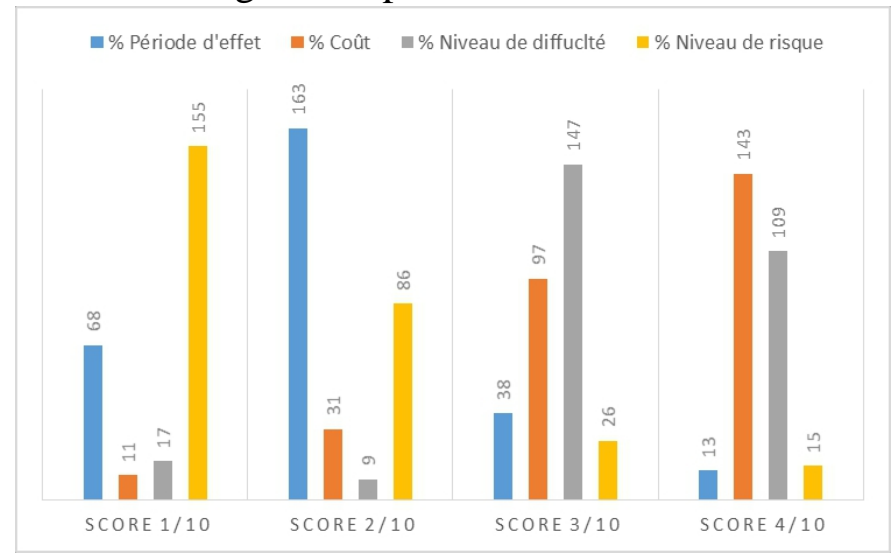

Figure 1 : Pondération des critères d'appréciation selon les producteurs enquêté

D'après cette figure :

- le critère "niveau de risque" est dominant dans la tranche de score $1 / 10$ : donc son poids selon les producteurs est 0,1 soit $10 \%$;

- le critère "période d'effet" est dominant dans la tranche de score $2 / 10$ : donc son poids selon les producteurs est 0,2 soit $20 \%$;

- le critère "niveau difficulté" qui renseigne sur la complexité de la technologie dans la mise en œuvre est dominant dans la tranche de score $3 / 10$ : donc son poids selon les producteurs est 0,3 soit $30 \%$;

- et le critère "'coût" qui renseigne sur la cherté de la technologie est dominant dans la tranche de score $4 / 10$ : donc son poids selon les producteurs est 0,4 soit $40 \%$.

Ces poids ont été utilisés dans la détermination de la valeur unique de l'alternative en vue d'ordonner les technologies selon la préférence dans chaque commune.

La valeur unique (score unique) provient de la somme pondérée des valeurs normées des critères par alternative. 


\section{Connaissance des technologies agro-écologiques}

Les producteurs enquêtés n'ont pas un même niveau de connaissance des différentes technologies agro-écologiques. Les techniques de conservation des sols qui prônent un travail minimum du sol sont très peu connues (strip till, zaï,). Il y en est de même des techniques agroforestières (Figure 2).

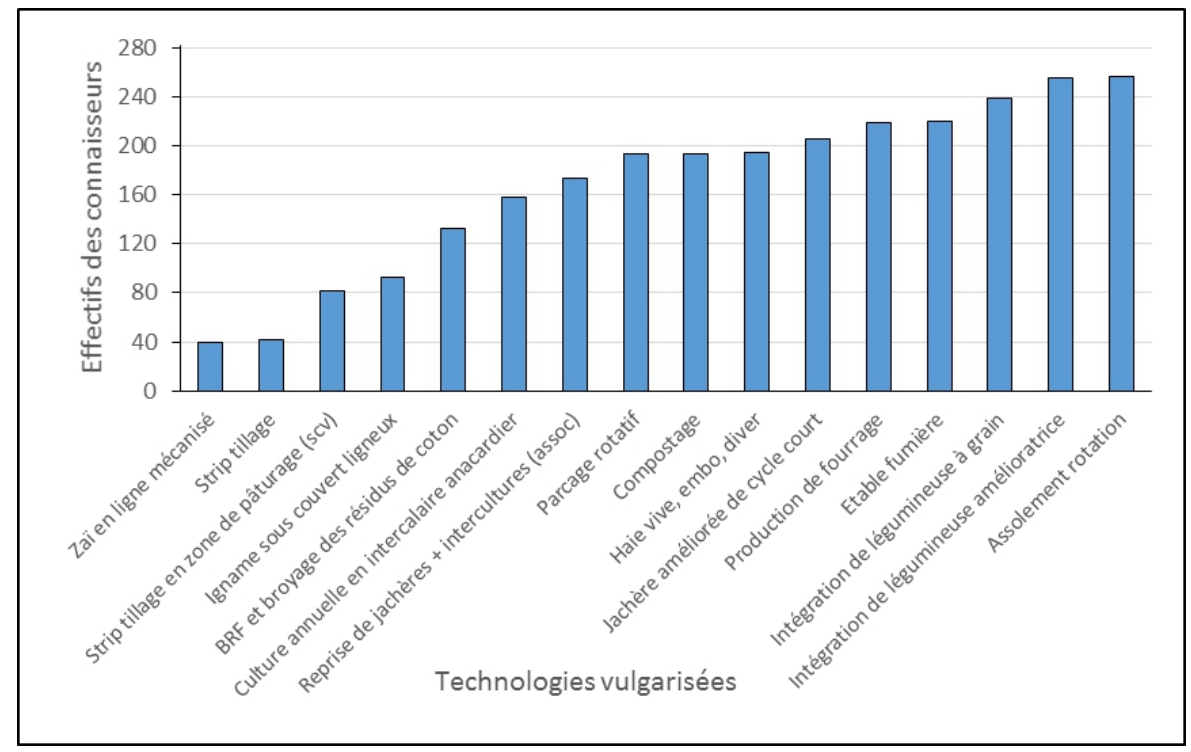

Figure 2 : Niveau de connaissance des techniques testées par les producteurs

\section{Pratique des technologies agro-écologiques}

Lors de l'étude, on s'est intéressé à connaître les technologies les plus testées par les producteurs. Les réponses favorables recueillies par technologie ont permis de tracer le graphe de la figure ci-dessous.

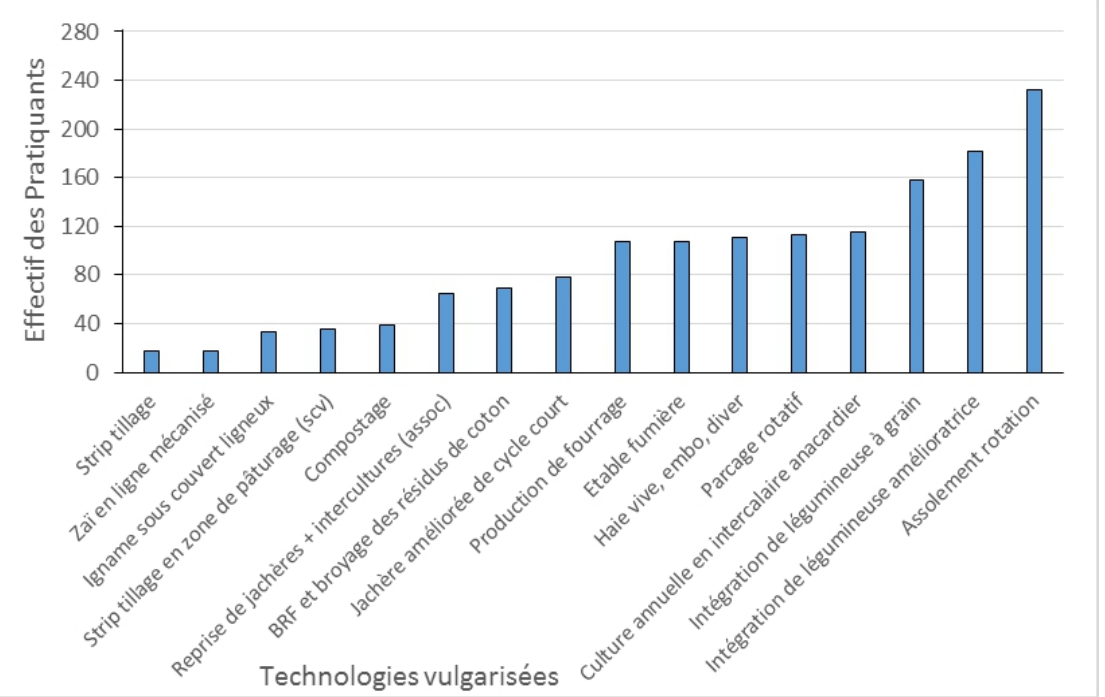

Figure 3 : Technologies testées par les producteurs de la zone d'étude 
A partir des effectifs recueillis, on peut distinguer trois niveau de technologies testées: les technologies bien testées (plus de 46\%), celles moyennement testées (35- 46\%) et les moins testées (0-35\%). Suivant ce classement on a: les technologies bien testées (assolement/rotation, intégration des légumineuses, culture en interligne des ligneux), celles moyennement testées (production fourragère, parcage rotatif, production de fumier) et les moins testées (compostage, le strip till, le zaï, les semis sous couvert végétal en reprise de jachère).

\section{Technologies appréciées par les pratiquants}

Les cinq alternatives les mieux appréciées par commune sont présentées dans le Tableau 3

Tableau 3 : Les cinq technologies plus préférées par commune

\begin{tabular}{c|l|l|l|l|l}
\hline $\begin{array}{l}\text { Ordre de } \\
\text { Préférence }\end{array}$ & Banikoara & Kandi & $\begin{array}{l}\text { Ouassa- } \\
\text { Péhunco }\end{array}$ & Parakou & Savalou \\
\hline 1 & $\begin{array}{l}\text { Etable } \\
\text { fumière }\end{array}$ & $\begin{array}{l}\text { Reprise de } \\
\text { jachères }+ \\
\text { inter cultures }\end{array}$ & $\begin{array}{l}\text { Production de } \\
\text { fourrage }\end{array}$ & $\begin{array}{l}\text { Intégration de } \\
\text { légumineuse } \\
\text { amélioratrice }\end{array}$ & $\begin{array}{l}\text { BRF et broyage des } \\
\text { résidus de coton }\end{array}$ \\
\hline 2 & $\begin{array}{l}\text { Parcage } \\
\text { rotatif }\end{array}$ & $\begin{array}{l}\text { Intégration } \\
\text { de } \\
\text { légumineuse } \\
\text { amélioratrice }\end{array}$ & $\begin{array}{l}\text { Intégration de } \\
\text { légumineuse } \\
\text { amélioratrice }\end{array}$ & $\begin{array}{l}\text { Jachère améliorée } \\
\text { de cycle court }\end{array}$ & Etable fumière \\
\hline 3 & $\begin{array}{l}\text { Haie vive et } \\
\text { embocage }\end{array}$ & $\begin{array}{l}\text { Production } \\
\text { de fourrage }\end{array}$ & Parcage rotatif & Parcage rotatif & Haie vive, embocage \\
\hline \multirow{2}{*}{5} & $\begin{array}{l}\text { Assolement/ } \\
\text { rotation }\end{array}$ & $\begin{array}{l}\text { Intégration } \\
\text { de } \\
\text { légumineuse } \\
\text { à grain }\end{array}$ & $\begin{array}{l}\text { Culture annuelle } \\
\text { en intercalaire } \\
\text { anacardier }\end{array}$ & $\begin{array}{l}\text { Reprise de jachères } \\
+ \text { inter cultures }\end{array}$ & Assolement/rotation \\
\hline & $\begin{array}{l}\text { Intégration } \\
\text { de } \\
\text { légumineuse } \\
\text { amélioratrice }\end{array}$ & $\begin{array}{l}\text { Parcage } \\
\text { rotatif }\end{array}$ & $\begin{array}{l}\text { Haie vive, } \\
\text { embocage }\end{array}$ & Etable fumière & Parcage rotatif \\
\hline
\end{tabular}

On s'aperçoit que l'ordre de préférence n'est pas le même allant d'une commune à l'autre. Mais les technologies de la famille dite 'Gestion Intégrée de la Fertilité des Sols (GIFS) dominent les choix, suivies des technologies de la famille 'Gestion Intégrée Agriculture/Elevage (GIAE).

Pour bien expliquer les préférences des expérimentateurs, les niveaux de pratiques des technologies ont été rapportés à leur niveau de connaissance.

\section{Connaissance des technologies pratiquées}

On a remarqué comme le montre la figure 4 que les producteurs enquêtés n'avaient pas une bonne connaissance de toutes les technologies 
vulgarisées. Ils connaissent mieux celles qu'ils pratiquent comme le montre la figure ci-dessous.

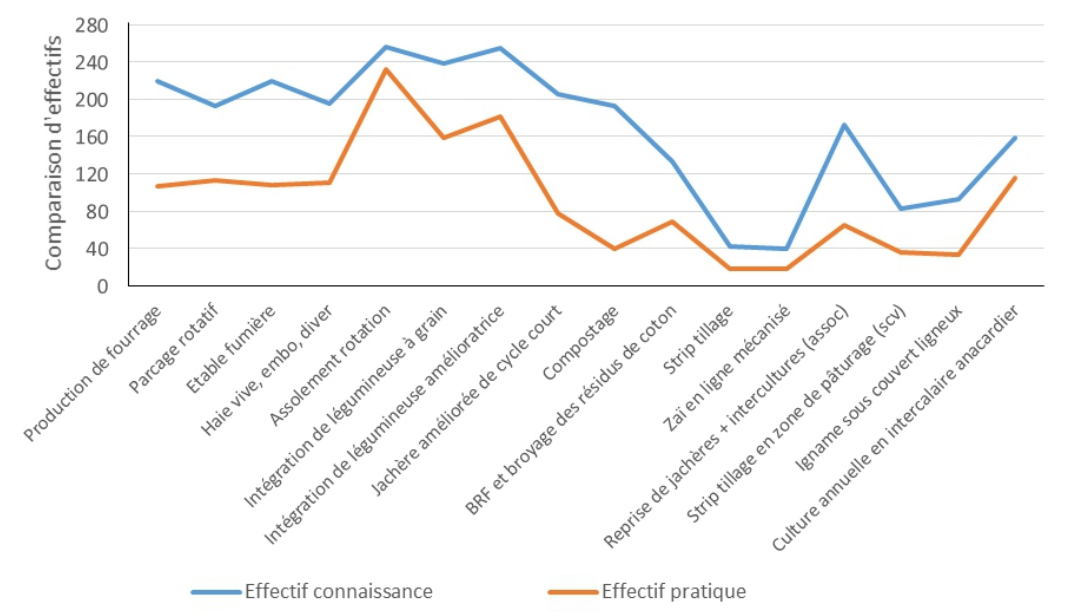

Figure 4 : Comparaison des courbes de connaissance et des pratiques des technologies agroécologiques

En mettant ensemble la courbe de connaissance des techniques agroécologiques et la courbe de leur mise en pratique (test) par les multiplicateurs, on constate que les deux courbes ont quasiment la même allure. Ce qui suppose une corrélation entre les deux données. En effet, on ne peut pratiquer que quand on connait. Et pour des décisions objectivement soutenables, les avis de ceux qui connaissent et qui pratiquent sont d'une grande importance.

\section{Discussion}

\section{Déterminants de la préférence des producteurs}

On note globalement que les technologies de la famille "gestion intégrée de la fertilité des sols » et celles de la famille "gestion intégrée agriculture-élevage» ont les meilleures cotes. Ce qui montre que les producteurs de la zone d'étude ont conscience du réel problème de la baisse de fertilité et ses conséquences sur leurs exploitations. Aussi, comme les producteurs élèvent et utilisent la force animale pour la réalisation de certains travaux champêtres, ils sont conscients de la nécessité de faire cohabiter les deux systèmes de productions. Pour cela ils apprécient ces deux familles de technologies qui valorisent les résidus de récolte et restaurent la fertilité du sol. Ce résultat corrobore celui de Djenontin et al. (2003) et de FAO (2008), qui ont montré l'importance de la valorisation des résidus de récolte et le système de parcage rotatif des animaux sur l'amélioration de la fertilité des sols. Les trois autres familles à savoir « l'agriculture de conservation», «la conservation des eaux et des sols » et «l'agroforesterie » ne sont pas trop pratiquées parce que mal connues. Cependant, il faut faire remarquer que ce 
résultat s'explique en partie par la méthodologie utilisée pour conduire le travail. En effet, le fait de choisir quelques technologies pour pratiquer a permis aux expérimentateurs de renforcer leur connaissance en ces technologies mais d'oublier celles non pratiquées. Or lors de l'enquête, chaque producteur est amené à se prononcer sur l'ensemble des technologies vulgarisées. Les meilleurs scores sont allés naturellement aux technologies les mieux pratiquées. Pour favoriser la bonne perception des autres technologies proposées par les cibles bénéficiaires, il faut donc mettre l'accent sur les techniques de diffusion des innovations telle que la démonstration. Des résultats obtenus, il ressort que les technologies pratiquées ne sont pas les mêmes, allant d'une commune à l'autre. On remarque que les techniques de la famille "gestion intégrée de la fertilité des sols » et celles de la famille «gestion intégrée agriculture-élevage» sont plus préférées dans les communes du Nord que dans celles du Sud du pays. Cette préférence est due aux systèmes de productions en vogue dans les différentes régions. Ce résultat corrobore ceux de Galliano et Nadel (2013) qui ont prouvé dans leurs travaux sur « Les déterminants de l'adoption de l'éco-innovation selon le profil stratégique de la firme : le cas des firmes industrielles françaises » que des caractéristiques externes telle que le système de production du milieu influence le choix des technologies. Comme le montre la figure 5, la production animale est plus prépondérante allant du nord vers le sud.

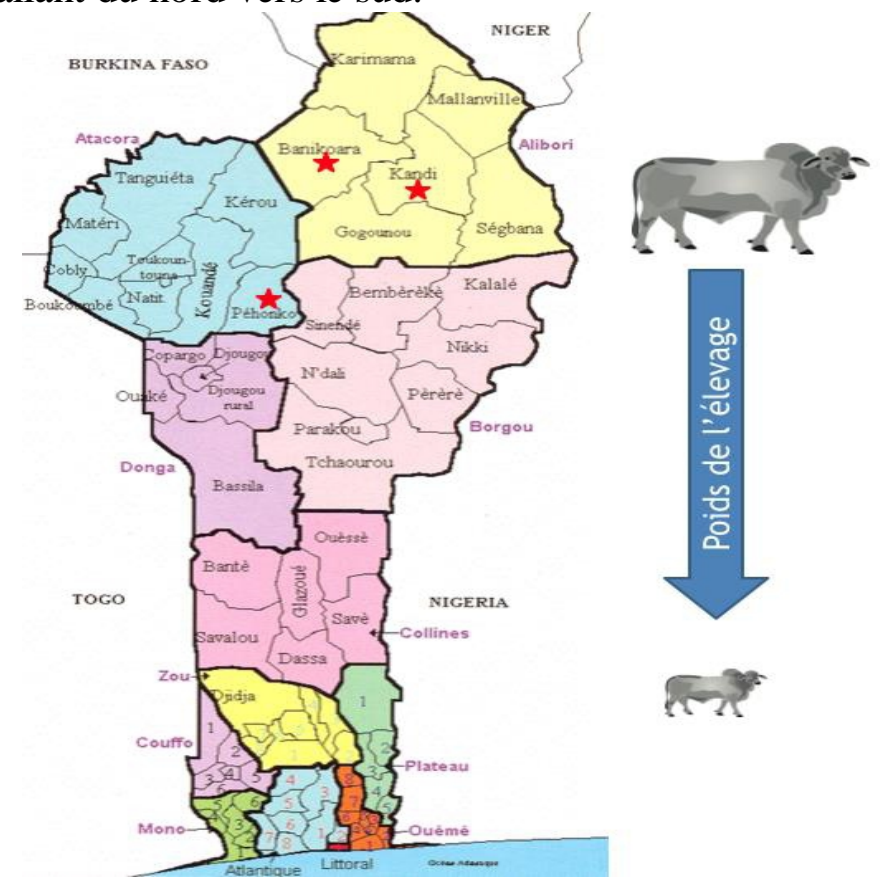

Figure 5 : Zones d'élevage en République du Bénin

Source : Rapport de diagnostic du projet TAZCO, 2017. 
Il y a donc plus d'animaux dans les communes de Kandi, Banikoara et Ouassa-Péhunco que dans les Communes de Parakou et Savalou qui sont au Sud de la zone de production cotonnière. Cela démontre combien il y a de pression sur les terres agricoles dans les communes du Nord de la zone cotonnière car, ces communes sont également les grandes communes de production et concentrent près de $2 / 3$ des superficies emblavées en culture de coton au Bénin par an. Ce résultat confirme ceux de Baco et al. (2007) qui ont montré l'ampleur de la pression foncière dans les zones cotonnières au Bénin. La cohabitation du grand cheptel qui est à la quête permanente de la vaine pâture avec les grandes superficies d'emblavure de cultures qui empiètent abondamment les espaces de pâturage engendrent de fréquents conflits entre agriculteurs et éleveurs. Donc l'espace pour cultiver et l'espace pour le pâturage du bétail sont des enjeux majeurs dont ont conscience les agriculteurs de ces zones. On peut donc affirmer que c'est fort de ces enjeux que les technologies GIFS qui permettent d'exploiter durablement et les technologies GIAE qui contribuent à la pacification des relations agriculteurs-éleveurs sont plus appréciées dans ces zones. Par contre dans les communes de Parakou et de Savalou où les emblavures sont moindres, la production de l'anacarde est en pleine expansion. Cela démontre bien pourquoi dans la liste restreinte des cinq technologies préférées dans ces zones apparait la famille de l'agroforesterie. Donc pour donner assez de chance à une technologie d'être adoptée dans une zone, il faut qu'elle soit une solution aux problèmes récurrents les plus perçus par les bénéficiaires. L'adoption rapide des pratiques agro-écologiques par les producteurs de céréale au Mali pour améliorer la fertilité des sols et s'adapter aux changements climatiques en est une parfaite illustration (Zoundji et al., 2018). Parmi les technologies les moins pratiquées par les expérimentateurs impactés par le projet TAZCO, il y a les technologies de la famille «conservation des eaux et des sols», celles de la famille "agriculture de conservation» et l'agroforesterie. Les deux premières familles ont pour principes un travail minimum des sols avec le maintien permanent du couvert végétal sur le sol. Travailler la terre en condition de couverture par des végétaux n'est pas aisé sans un niveau de mécanisation adaptée. La mécanisation étant encore d'un faible niveau et inapproprié dans l'agriculture béninoise, elle n'a permis aux producteurs d'aller vers les familles de technologies qui exigent une mécanisation spécifique. De plus quand le travail est difficile et complexe comme cultiver sur un sol couvert, la main d'œuvre nécessaire par unité de surface est élevée ainsi que les coûts à l'unité de surface. Pour cela, il est à souhaiter qu'une mécanisation adéquate à la typologie des exploitants et à aux terroirs soit développé pour améliorer l'acceptation des innovations dites de conservation.

Outils utilisés pour la détermination de préférence du groupe cible 
Du point de vue méthodologique, notons que le Front de Pareto n'a pas été utilisé dans son sens premier, dans le cadre de la présente étude. Dans son sens premier, le niveau de domination zéro signifie pour une alternative donnée qu'elle est meilleure à toutes les autres sur au moins un des critères et représente donc une alternative à prendre en compte. Les autres sont ainsi rejetées parce que dominées. Pris dans ce sens, il n'y avait qu'une ou deux technologies par commune; ce qui ne serait pas du goût des dirigeants du projet qui veulent plus de stratégies à proposer. En reprenant les alternatives peu dominées dans ce postulat, on a pu obtenir le nombre de cinq alternatives souhaitées par les dirigeants du projet. Sans ce premier tri avec le Front de Pareto, il serait possible d'aboutir au classement en ordre des alternatives en combinant la fonction de désirabilité et le critère unique pour dégager les cinq meilleures voulues. Mais dans ce cas, avec toute la liste des alternatives. Or cette liste est de dix-sept ; elle pouvait être plus et être davantage plus difficile. C'est pour contourner cette difficulté que le Front de Pareto a été utilisé pour restreindre la liste en éliminant d'abord les moins pertinentes. Si la difficulté de longue liste n'est pas bien appréhendée dans le cas actuel, elle peut l'être avec une liste de cent ou deux cent alternatives que le Front de Pareto aiderait à réduire en l'utilisant de la manière proposée dans le cadre de cette étude.

La fonction de désirabilité a été utilisée pour donner un sens au score recueilli par chaque alternative suite à l'appréciation des producteurs. En effet, le score (1, 2 ou 3) en lui-même n'est pas expressif du souhait du producteur qui apprécie. Il y a des cas où c'est la valeur moindre qui est souhaitée et il y a d'autres où c'est la valeur plus importante qui est souhaitée. Par exemple quand on demande au producteur de donner une note par rapport au temps mis par une technologie (une alternative) pour améliorer la fertilité du sol, il attribue : $0=$ ne sait pas ; $1=$ court; $2=$ moyen et $3=$ trop long. Par contre lorsqu'on lui demande le niveau de gain associé, il attribue : $0=$ ne sait pas ; 1= bas ; 2= moyen et 3= élevé. La valeur 0 est neutre dans tous les cas parce qu'elle exprime une situation indécise. Par contre les autres n'expriment pas la même chose dans tous les cas. Dans l'exemple exposé, il préfère la technologie (alternative) qui recueille dans le premier cas 1 qui signifie amélioration de la fertilité en un délai court tandis qu'il déteste le 1 du second cas qui signifie que le niveau de gain associé est bas. Inversement, il apprécie le niveau de gain élevé $=3$ et déteste $3=$ long temps mis pour améliorer la fertilité du sol. Dans le processus de normalisation des valeurs, cet aspect est pris en compte par les formules qui précisent si le critère est à minimiser ou à maximiser. Chaque valeur est rapportée à la situation désirée.

En ce qui concerne le critère unique, il agrège les valeurs des différents critères au niveau d'une même alternative en une seule valeur d'appréciation. Autrement on serait obligé d'analyser les alternatives, critère par critère. 


\section{Conclusion}

Il ressort de la présente que les pratiques agro-écologiques vulgarisées par le projet TAZCO sont plus ou moins bien connues par les producteurs de la zone cible. Cependant, on note que ces producteurs se rappellent seulement d'un nombre limité de technologies agro-écologiques (entre 5 et 6). Les technologies dont ils se souviennent le plus sont celles qui ont intégré les pratiques culturales de la zone à cause de leur faible niveau de complexité, leur faible dépendance en équipement spécifiques et ayant un intérêt autre que celle de la seule restauration des sols. D'autres technologiques potentiellement bénéfiques (strip-till, zaï en ligne, broyage de résidus, aménagement en courbe de niveau, etc.) n'ont pas été adoptées pour des raisons de manque de connaissance et de pratique. On note également une émergence des pratiques agroforestières telles que la culture annuelle sous anacardier à cause de l'anacarde qui prend de la valeur depuis quelques années, la préservation des arbres de Karité dans les exploitations à cause des noix que collectent les femmes pour l'extraction du beurre qui est très consommée dans le nordBénin, etc. Ces pratiques agroforestières ne sont pas expressément citées parmi les technologies agro-écologiques préférées des producteurs. Le fait pour le producteur de pratiquer l'agroécologie sans en avoir conscience et de ne pas préférer une technologique qui lui est potentiellement bénéfique montre que la préférence d'une technologie par le producteur dépend plus de l'intérêt qu'il perçoit dans sa mise en œuvre que de celui qu'on lui miroite.

Donc pour attirer la conscience des producteurs vers une technologie donnée, il faut :

- intensifier la démonstration avec des moyens économiquement et socialement accessible ;

- travailler à minimiser les coûts d'accès aux équipements en encourageant la reproduction locale qui pourrait rendre les machines agricoles disponibles à moindre coûts ;

- faire des concertations pour éviter que les efforts d'adoption des pratiques agro-écologiques par certains exploitants ne soient découragés par les mauvaises pratiques d'autres exploitants (divagation de bêtes dans une parcelle en préparation pour semis sous couvert végétal la campagne suivante, les feux de brousse criminels, etc.).

\section{References:}

1. Adégbola, Y., P., Oussou, B., T., C., Adoko, K., F., \&Crinot, F., G. (2016). Analysis of research and innovations labors on the sustainable management of lands in Benin. Information and technical sheet $\mathrm{N}^{\circ} 2$. Legal Deposit $N^{\circ} 8731$ of June $17^{\text {th }}, 2013$, National Library of Benin. ISBN : 978-99919-2-301-7. 
2. Adegbola, P., \&Adekambi, S. (2006). Impact socio-économique de l'adoption des technologies nouvelles de gestion de la fertilité des sols au Bénin. Cas des jachères Mucuna, acacia, aeschynomene et glyricidia. Rapport d'étude. INRAB/MAEP.

3. Adesina, A., A., \&Zinnah, M., M. (1993). Technology characteristics, farmers' perceptions and adoption decisions: A Tobit model application in Sierra Leone. Agricultural Economics 9(4): 297-311.

4. Alohou, E., \&Agossou, V. (1998). Diagnostic sur la gestion des sols : cas de Kokey et de Bensékou. Rapport d'étude.

5. Akpinfa, E. D., Kissira, A., Akpo, M., A., \& Houssou, C. S. (2017). Evaluation du coût économique de la dégradation des terres dans la zone agro-écologique du centre Bénin. EuropeanScientific Journal, 13 (6): 1857 - 7881.

6. Akomagni, L., A., \&Guidibi, E. (2006). Monographie de la Commune de Parakou. Rapport Afrique Conseil.

7. Anderson, W., K., \&Siddiqu, K. H. M. (2015). The role and value of crop residues in dryland agriculture. Indian Journal of Agronomy60 (3): 332-340.

8. Baco, N., M., Djenontin, J., A., \&Amidou, M. (2007). Gestion de la fertilité des sols dans le nord du Bénin et incidences économiques pour les exploitations agricoles. 2003. hal-00133339.

9. Benki, A. (2014). Méthodes efficaces de capture de front de pareto en conception mécanique multicritère : applications industrielles. Mathématiques générales, Université Nice Sophia Antipolis, 2014. Français. NNT : 2014 NICE4000.

10. Berton, S., Billaz, R., Burger, P., \& Lebreton, A. (2013). Agroécologie, une transition vers des modes de vie et de développement viables - Paroles d'acteurs. Groupe de Travail Désertification (GTD), Janvier 2013.

11. Blaimont, A. (2013). Etude de la perception de l'innovation agroécologique en Wallonie : le cas de l'agroforesterie tempérée dans la Botte du Hainaut et le Brabant Wallon. Mémoire de fin d'études présenté en vue de l'obtention du diplôme de Master en Bioingénieur : Sciences et technologies de l'environnement. Université Libre de Bruxelles, Belgique.

12. CARDER, (2013). Description de la commune, bilan diagnostic et problématique de développement de la commune de Parakou, Rapport du Centre Agricole Régional pour le Développement Rural (CARDER) Borgou-Alibori.

13. Cheikho, A. (2015). L'adoption des innovations technologiques par les clients et son impact sur la relation client - Cas de la banque mobile. Université Nice Sophia Antipolis, 2015. 
14. Deniau C., Gaillard T., Mbagogo A., Réounodji B. M., Le Bel S. (2017). Using the KoBoCollect tool to analyze the socio-economic and socio-cultural aspects of commercial hunting and consumption of migratory waterbirds in the Lakes Chad and Fitri (Chad). in the Lakes Chad and Fitri (Chad). IRSTEA. N ISBN: 978-2-85362-686-6.

15. Djenontin, J., A., Amidou, M., \&Wennink, B. (2003). Valorisation des résidus de récolte dans l'exploitation agricole au nord du Bénin : production de fumier dans le parc de stabulation des bœufs. 2003, 8 p. hal-00137951.

16. Ekionea, J., P., B., Bernard, P., \& Plaisent, M. (2011). Consensus par la méthode Delphi sur les concepts clés des capacités organisationnelles spécifiques de la gestion des connaissances. Recherches Qualitatives, 29(3) 168-192.

17. FAO. (2014). L’agroécologie pour la sécurité alimentaire et la nutrition. Compte rendu du symposium international de la FAO 18-19 septembre 2014, Rome, Italie.

18. FAO. (2011). La pratique de la gestion durable des terres. Directives et bonnes pratiques pour l'Afrique subsaharienne ; applications sur le terrain.

19. FAO. (2008). Investing in Sustainable Agricultural Intensification, the role of Conservation Agriculture. Part III - a framework for action. An international technical workshop investing in sustainable crop intensification: The case for improving soil health, FAO, Rome: 22-24 July 2008. Integrated Crop Management, 6-2008.

20. Galliano, D., \&Nadel, S. (2013). Les déterminants de l'adoption de l'éco-innovation selon le profil stratégique de la firme : le cas des firmes industrielles françaises. Revue Economique et Industrielle $2^{\text {ème }}$ trimestre 2013, 77-110.

21. Guerin, J. (2017). Diagnostic agropastoral pour une transition agroécologique dans la zone cotonnière du Bénin : étude de cas dans trois terroirs villageois de la commune de Savalou.

22. Guillot-Soulez, C., \& Soulez, S. (2011). L'analyse conjointe : présentation de la méthode et potentiel d'application pour la recherche en GRH ; Revue de Gestion des Ressources Humaines ; SP33-EP 44 ; DO 10.3917/grhu.080.0033.

23. Igué, A., M., Adjanohoun, A., Saidou, A., Ezui, G., Attiogbe, P., Kpagbin, G., Gotoechan-Hodonou, H., Youl, S., Pare, T., Balogoun, I., Ouedraogo, J., Dossa, E., Mando, A., \&Sogbedji, J., M. (2013). Application et adaptation de l'approche intégrée DSSAT-SIG à la formulation des doses d'engrais pour la culture du maïs au Sud et au Centre du Bénin. Bulletin de la Recherche Agronomique du Bénin (BRAB) Numéro spécial Fertilité, 2013. 1840-7099. 
24. INSAE. (2016). Effectifs de la population des villages et Quartiers de ville du Benin (RGPH-4 2013), Cotonou.

25. Leterme, P., \& Morvan, T. (2010) Mieux valoriser la ressource dans le cadre de l'intensification écologique. Les colloques de l'Académie d'Agriculture de France, 1: 101-118.

26. MAEP, (2017). Plan Stratégique du Développement du Secteur Agricole (PSDSA), Ministère de l'Agriculture, de l'Elevage et de la Pêche, MAEP, Bénin.

27. MacLeod, N., D., \& McIvor J., G. (2005). Analysis reconciling economic and ecological conflicts for sustained management of grazing lands. Ecological Economics 56 (2006) 386- 401.

28. Menapace, L., Colson, G., \&Raffaelli, R. (2013). Risk Aversion, Subjective Beliefs, and Farmer Risk Management Strategies. American Journal of Agricultural Economics 95(2): 384-389.

29. Nougbodohoué, R. (2017). Diagnostic agropastoral et transition agroécologique des zones cotonnières du Bénin: Etude de cas de la commune de Ouassa-Péhunco.

30. Savadogo, M., Somda, J., Seynou, O., Zabré, S., Nianogo, A., J. (2011). Catalogue des Bonnes Pratiques d'Adaptation aux Risques Climatiques au Burkina Faso. UICN: Ouagadougou, Burkina Faso; 52.

31. Sermé, I., Outtara, K., Logah, V., Taounda, J., B., Pale, S., Quansah, C., Abaidoo, R. (2015). Impact of tillage and fertility management options on selected soil physical properties and sorghum yield. Int. J. Biol. Chem. Sci., 9 (3): 1154-1170. doi.org/10.4314/ijbcs.v9i3.2.

32. Shapiro, B., Brorsen, B., \& Doster, D. (1992). Adoption of doublecropping soybeans and wheat. Southern Journal of Agricultural Economics 24: 33-40.

33. Sherin, S. P., N, Mathew, P, Johns, F., Abraham, J. (2018). The feasibility of using remote data collection tools in field surveys. Int J Community Med Public Health2018; 5:81-5.

34. Sinsin, B., \& Kampmann, D. (2013). Atlas de la biodiversité de l'Afrique de l'Ouest, Tome I: Bénin, Institute of Physical Geography F.U., Federal Ministry of Education and Research (BMBF).

35. Vodouhê, F., G., Coulibaly, O., Adégbidi, A., \&Sinsin, B. (2010). Community perception of biodiversity conservation within protected areas in Benin. Forest Policy and Economics 12:505-512.

36. Zoundji, C., G., Vodouhê, D., S., Okry, F., Bentley, J. W., \& Tossou, R., C. (2018). Beyond Striga Management: Learning Videos Enhanced Farmers' Knowledge on Climate-Smart Agriculture in Mali. Sustainable Agriculture Research, Vol. 7, No. 1; 2018. doi:10.5539/sar.v7n1p80. 
Annexe: Démarche de détermination des cinq alternatives souhaitées par commune et leur hiérarchisation

\section{a. Cas de Banikoara}

Tableau 4 : Tri des technologies les plus préférées à Banikoara selon le Front de Pareto

\begin{tabular}{|c|c|c|c|c|c|}
\hline Alternatives & $\begin{array}{l}\text { Période de } \\
\text { fertilisatio } \\
\mathrm{n} \text { associée }\end{array}$ & $\begin{array}{l}\text { Coû } \\
\mathrm{t}\end{array}$ & $\begin{array}{l}\text { Niveau } \\
\text { de } \\
\text { difficult } \\
\text { é }\end{array}$ & $\begin{array}{l}\text { Nivea } \\
\text { u de } \\
\text { risque }\end{array}$ & $\begin{array}{c}\text { Solutions } \\
\text { dominante } \\
\text { S }\end{array}$ \\
\hline Parcage rotatif & 106 & 146 & 190 & 144 & 1 \\
\hline Etable fumière & 123 & 153 & 194 & 151 & 0 \\
\hline Haie vive et embocage & 37 & 140 & 192 & 145 & 2 \\
\hline Assolement/ rotation & 110 & 141 & 190 & 147 & 1 \\
\hline $\begin{array}{l}\text { Intégration de légumineuse } \\
\text { amélioratrice }\end{array}$ & 68 & 162 & 211 & 158 & 0 \\
\hline
\end{tabular}

Tableau 5 : Normalisation des valeurs

\begin{tabular}{|c|c|c|c|c|c|}
\hline \multicolumn{2}{|c|}{ Paramètres / alternatives } & $\begin{array}{l}\text { Période de } \\
\text { fertilisation associée }\end{array}$ & $\begin{array}{l}\text { Coût } \\
(0,4)\end{array}$ & $\begin{array}{l}\text { Niveau de } \\
\text { difficulté }\end{array}$ & $\begin{array}{l}\text { Niveau } \\
\text { de risque }\end{array}$ \\
\hline \multirow{2}{*}{$\begin{array}{l}\text { Paramètre } \\
\text { des } \\
\text { fonctions }\end{array}$} & Seuil bas & 37 & 140 & 190 & 144 \\
\hline & Seuil haut & 123 & 162 & 211 & 158 \\
\hline \multirow{5}{*}{$\begin{array}{l}\text { Valeurs } \\
\text { normées }\end{array}$} & Parcage rotatif & 0,80 & 0,27 & 1 & 1 \\
\hline & Etable fumière & 1 & 0,59 & 0,81 & 0,5 \\
\hline & $\begin{array}{l}\text { Haie vive et } \\
\text { embocage }\end{array}$ & 0 & 0 & 0,905 & 0,93 \\
\hline & Assolement/ rotation & 0,85 & 0,04 & 1 & 0,79 \\
\hline & $\begin{array}{l}\text { Intégration de } \\
\text { légumineuse } \\
\text { amélioratrice }\end{array}$ & 0,36 & 1 & 0 & 0 \\
\hline
\end{tabular}

Leur réduction en une valeur unique de comparaison donne :

Tableau 6: Critère unique et ordre de préférence des alternatives

\begin{tabular}{l|c|c}
\hline Alternatives & Score unique & Rang \\
\hline Parcage rotatif & $0,05 \%$ & 2 \\
Etable fumière & $0,06 \%$ & 1 \\
Haie vive et embocage & $0,00 \%$ & 4 \\
Assolement/ rotation & $0,01 \%$ & 3 \\
Intégration de légumineuse amélioratrice & $0,00 \%$ & 5 \\
\hline
\end{tabular}

Par exemple le premier score unique : $0,05252 \%=(0,2 * 0,802325581) \mathrm{x}$ $(0,4 * 0,272727273) \times\left(0,3^{*} 1\right) \times(0,1 * 1)$.

\section{b. Cas de Kandi}

$\underline{\text { Tableau } 7}$ : Tri des technologies plus préférées à Kandi selon le Front de Pareto 


\begin{tabular}{l|c|c|c|c|c}
\cline { 2 - 6 } Alternatives & $\begin{array}{l}\text { Période } \\
\text { d'effet }\end{array}$ & Coût & $\begin{array}{l}\text { Niveau } \\
\text { de } \\
\text { difficulté }\end{array}$ & $\begin{array}{l}\text { Niveau } \\
\text { de } \\
\text { risque }\end{array}$ & $\begin{array}{l}\text { Solutions } \\
\text { dominantes }\end{array}$ \\
\hline Production de fourrage & 103 & 112 & 158 & 124 & 2 \\
Parcage rotatif & 108 & 83 & 128 & 99 & 2 \\
Assolement rotation & 132 & 137 & 197 & 156 & 0 \\
Intégration de légumineuse à grain & 87 & 109 & 153 & 128 & 2 \\
Intégration de légumineuse amélioratrice & 109 & 116 & 162 & 132 & 1 \\
Jachère améliorée de cycle court & 85 & 115 & 164 & 132 & 1 \\
Reprise de jachères + inter cultures & 104 & 103 & 144 & 84 & 2 \\
\hline
\end{tabular}

Tableau 8 : Normalisation des valeurs

\begin{tabular}{|c|c|c|c|c|c|}
\hline & \multirow{3}{*}{$\begin{array}{c}\begin{array}{c}\text { Période } \\
\text { de d'effet }\end{array} \\
85\end{array}$} & \multirow{3}{*}{$\begin{array}{c}\text { Coût } \\
83\end{array}$} & \multirow[b]{2}{*}{$\begin{array}{c}\text { Niveau } \\
\text { de } \\
\text { difficulté }\end{array}$} & \multirow[b]{2}{*}{$\begin{array}{c}\text { Niveau } \\
\text { de } \\
\text { risque }\end{array}$} \\
\hline & & & & & \\
\hline Paramètre & Seuil bas & & & 128 & 84 \\
\hline fonctions & Seuil haut & 132 & 137 & 197 & 156 \\
\hline \multirow{7}{*}{$\begin{array}{l}\text { Valeurs } \\
\text { normées }\end{array}$} & Production de fourrage & 0,38 & 0,54 & 0,56 & 0,44 \\
\hline & Parcage rotatif & 0,49 & 0 & 1 & 0,79 \\
\hline & Assolement rotation & 1 & 1 & 0 & 0 \\
\hline & $\begin{array}{l}\text { Intégration de légumineuse à } \\
\text { grain }\end{array}$ & 0,04 & 0,48 & 0,64 & 0,39 \\
\hline & $\begin{array}{l}\text { Intégration de légumineuse } \\
\text { amélioratrice }\end{array}$ & 0,51 & 0,61 & 0,51 & 0,33 \\
\hline & Jachère améliorée de cycle court & 0 & 0,59 & 0,48 & 0,33 \\
\hline & $\begin{array}{l}\text { Reprise de jachères + inter } \\
\text { cultures }\end{array}$ & 0,40 & 0,37 & 0,77 & 1 \\
\hline
\end{tabular}

Tableau 9 : Critère unique et ordre de préférence des alternatives

\begin{tabular}{l|c|c}
\cline { 2 - 3 } Alternatives & Score unique & Rang \\
\hline Production de fourrage & $0,01 \%$ & 3 \\
Parcage rotatif & $0,00 \%$ & 5 \\
Assolement rotation & $0,00 \%$ & 6 \\
Intégration de légumineuse à grain & $0,001 \%$ & 4 \\
Intégration de légumineuse amélioratrice & $0,01 \%$ & 2 \\
Jachère améliorée de cycle court & $0,00000 \%$ & 7 \\
Reprise de jachères + inter cultures & $0,02760 \%$ & 1 \\
\hline
\end{tabular}

\section{c. Cas de Ouassa-Péhunco}

Tableau 10 : Tri des technologies plus préférées à Oussa-Péhunco selon le Front de Pareto

Alternatives

Période d'effet

Coût

\begin{tabular}{|l|l|}
\hline $\begin{array}{l}\text { Niveau } \\
\text { de }\end{array}$ & $\begin{array}{l}\text { Niveau } \\
\text { de } \\
\text { difficulté }\end{array}$ \\
risque
\end{tabular}




\begin{tabular}{l|c|c|c|c|c}
\hline Production de fourrage & 91 & 118 & 154 & 123 & 1 \\
Parcage rotatif & 84 & 105 & 147 & 111 & 2 \\
Haie vive, embocage & 59 & 85 & 121 & 92 & 4 \\
Assolement/ rotation & 123 & 152 & 200 & 150 & 0 \\
$\begin{array}{l}\text { Intégration de légumineuse } \\
\text { amélioratrice }\end{array}$ & 87 & 104 & 141 & 109 & 2 \\
$\begin{array}{l}\text { Culture annuelle en } \\
\text { intercalaire anacardier }\end{array}$ & 67 & 88 & 128 & 91 & 4 \\
\hline
\end{tabular}

Tableau 11 : Normalisation des valeurs

\begin{tabular}{|c|c|c|c|c|c|}
\hline & & & & & \\
\hline & & $\begin{array}{l}\text { Période } \\
\text { d'effet }\end{array}$ & Coût & $\begin{array}{l}\text { Niveau de } \\
\text { difficulté }\end{array}$ & $\begin{array}{l}\text { Niveau de } \\
\text { risque }\end{array}$ \\
\hline \multirow{2}{*}{$\begin{array}{l}\text { Paramètre } \\
\text { des fonctions }\end{array}$} & Seuil bas & 59 & 85 & 121 & 91 \\
\hline & Seuil haut & 123 & 152 & 200 & 150 \\
\hline \multirow{6}{*}{$\begin{array}{l}\text { Valeurs } \\
\text { normées }\end{array}$} & Production de fourrage & 0,50 & 0,49 & 0,58 & 0,46 \\
\hline & Parcage rotatif & 0,39 & 0,30 & 0,67 & 0,66 \\
\hline & Haie vive, embocage & 0 & 0 & 1 & 0,98 \\
\hline & Assolement/ rotation & 1 & 1 & 0 & 0 \\
\hline & $\begin{array}{l}\text { Intégration de légumineuse } \\
\text { amélioratrice }\end{array}$ & 0,44 & 0,28 & 0,75 & 0,69 \\
\hline & $\begin{array}{l}\text { Culture annuelle en intercalaire } \\
\text { anacardier }\end{array}$ & 0,12 & $\begin{array}{c}0,04 \\
5\end{array}$ & 0,91 & 1 \\
\hline
\end{tabular}

$\underline{\text { Tableau } 12}$ : Critère unique et ordre de préférence des alternatives

\begin{tabular}{l|c|c}
\cline { 2 - 3 } & Score unique & Rang \\
\hline Production de fourrage & $0,016 \%$ & 1 \\
Parcage rotatif & $0,012 \%$ & 3 \\
Haie vive, embocage & $0,00 \%$ & 5 \\
Assolement/ rotation & $0,00 \%$ & 6 \\
Intégration de légumineuse amélioratrice & $0,015 \%$ & 2 \\
Culture annuelle en intercalaire anacardier & $0,001 \%$ & 4 \\
\hline
\end{tabular}

\section{d. Cas de Parakou}

Tableau 13 : Tri des technologies plus préférées à Parakou selon le Front de Pareto

\begin{tabular}{l|c|c|c|c|c}
\cline { 2 - 6 } Technologie & $\begin{array}{l}\text { Période } \\
\text { d'effet }\end{array}$ & Coût & $\begin{array}{l}\text { Niveau de } \\
\text { difficulté }\end{array}$ & $\begin{array}{l}\text { Niveau de } \\
\text { risque }\end{array}$ & $\begin{array}{l}\text { Solutions } \\
\text { dominantes }\end{array}$ \\
\hline Parcage rotatif & 38 & 47 & 68 & 55 & 2 \\
Etable fumière & 44 & 38 & 49 & 55 & 2 \\
Assolement/ rotation & 106 & 128 & 174 & 130 & 0 \\
Intégration de légumineuse à grain & 23 & 50 & 55 & 54 & 2 \\
Intégration de légumineuse amélioratrice & 50 & 81 & 110 & 93 & 1 \\
Jachère améliorée de cycle court & 36 & 51 & 61 & 51 & 2
\end{tabular}


Reprise de jachères + inter cultures 35 45 76 2

Tableau 14 : Normalisation des valeurs

\begin{tabular}{|c|c|c|c|c|c|}
\hline & $\begin{array}{l}\text { Période } \\
\text { d'effet }\end{array}$ & Coût & $\begin{array}{l}\text { Niveau de } \\
\text { difficulté }\end{array}$ & $\begin{array}{l}\text { Niveau } \\
\text { de risque }\end{array}$ \\
\hline Paramètre & Seuil bas & 23 & 38 & 49 & 51 \\
\hline $\begin{array}{c}\text { des } \\
\text { fonctions }\end{array}$ & Seuil haut & 106 & 128 & 174 & 130 \\
\hline \multirow{7}{*}{$\begin{array}{l}\text { Valeurs } \\
\text { normées }\end{array}$} & Parcage rotatif & 0,18 & 0,1 & 0,848 & 0,95 \\
\hline & Etable fumière & 0,25 & 0 & 1 & 0,95 \\
\hline & Assolement/ rotation & 1 & 1 & 0 & 0 \\
\hline & $\begin{array}{l}\text { Intégration de légumineuse à } \\
\text { grain }\end{array}$ & 0 & 0,13 & 0,952 & 0,96 \\
\hline & $\begin{array}{l}\text { Intégration de légumineuse } \\
\text { amélioratrice }\end{array}$ & 0,32 & 0,48 & 0,512 & 0,47 \\
\hline & Jachère améliorée de cycle court & 0,16 & 0,14 & 0,904 & 1 \\
\hline & $\begin{array}{l}\text { Reprise de jachères + inter } \\
\text { cultures }\end{array}$ & 0,14 & 0,08 & 0,784 & 1 \\
\hline
\end{tabular}

Tableau 15 : Critère unique et ordre de préférence des alternatives

\begin{tabular}{l|c|c}
\cline { 2 - 3 } Technologies & Score unique & Rang \\
\hline Parcage rotatif & $0,003 \%$ & 3 \\
Etable fumière & $0,00 \%$ & 5 \\
Assolement/ rotation & $0,00 \%$ & 6 \\
Intégration de légumineuse à grain & $0,00 \%$ & 7 \\
Intégration de légumineuse amélioratrice & $0,009 \%$ & 1 \\
Jachère améliorée de cycle court & $0,005 \%$ & 2 \\
Reprise de jachères + inter cultures & $0,002 \%$ & 4 \\
\hline
\end{tabular}

\section{e. Cas de Savalou}

Tableau 16: Tri des technologies plus préférées à Savalou selon le Front de Pareto

\begin{tabular}{l|c|c|c|c|c}
\cline { 2 - 6 } Technologies & $\begin{array}{l}\text { Période } \\
\text { d'effet }\end{array}$ & $\begin{array}{l}\text { Co } \\
\text { ût }\end{array}$ & $\begin{array}{l}\text { Niveau de } \\
\text { difficulté }\end{array}$ & $\begin{array}{l}\text { Niveau de } \\
\text { risque }\end{array}$ & $\begin{array}{c}\text { Solutions } \\
\text { dominantes }\end{array}$ \\
\hline Assolement/rotation & 48 & 69 & 101 & 87 & 1 \\
Parcage rotatif & 112 & 94 & 146 & 118 & 0 \\
Etable fumière & 73 & 62 & 101 & 87 & 1 \\
Haie vive, embocage & 48 & 54 & 77 & 60 & 4 \\
$\begin{array}{l}\text { BRF et broyage des résidus } \\
\text { de coton }\end{array}$ & 107 & 95 & 132 & 80 & 0 \\
$\begin{array}{l}\text { Culture annuelle en } \\
\text { intercalaire anacardier }\end{array}$ & 10 & 45 & 78 & 60 & 4 \\
\hline
\end{tabular}


$\underline{\text { Tableau } 17}$ : Normalisation des valeurs

\begin{tabular}{|c|c|c|c|c|c|}
\hline & & & & \multirow{3}{*}{$\begin{array}{c}\begin{array}{l}\text { Niveau de } \\
\text { difficulté }\end{array} \\
77\end{array}$} & \multirow{3}{*}{\begin{tabular}{|c|}
$\begin{array}{l}\text { Niveau } \\
\text { de risque }\end{array}$ \\
60
\end{tabular}} \\
\hline & \multirow{2}{*}{$\begin{array}{l}\begin{array}{l}\text { Période } \\
\text { d'effet }\end{array} \\
10\end{array}$} & \multirow{2}{*}{$\begin{array}{r}\text { Coût } \\
45\end{array}$} & & \\
\hline $\begin{array}{l}\text { Paramèt } \\
\text { re des }\end{array}$ & Seuil bas & & & & \\
\hline $\begin{array}{c}\text { fonction } \\
\qquad \mathrm{S}\end{array}$ & Seuil haut & 112 & 94 & 146 & 118 \\
\hline \multirow{6}{*}{$\begin{array}{c}\text { Valeurs } \\
\text { normée } \\
\text { s }\end{array}$} & Assolement/rotation & 0,37 & 0,49 & 0,65 & 0,53 \\
\hline & Parcage rotatif & 1 & 1 & 0 & 0 \\
\hline & Etable fumière & 0,68 & 0,35 & 0,65 & 0,53 \\
\hline & Haie vive, embocage & 0,37 & 0,18 & 1 & 1 \\
\hline & $\begin{array}{l}\text { BRF et broyage des } \\
\text { résidus de coton }\end{array}$ & 0,95 & 1 & 0,20 & 0,65 \\
\hline & $\begin{array}{l}\text { Culture annuelle en } \\
\text { intercalaire anacardier }\end{array}$ & 0 & 0 & 0,98 & 1 \\
\hline
\end{tabular}

Tableau 18 : Critère unique et ordre de préférence des alternatives

Technologies

Assolement/rotation

Parcage rotatif

Etable fumière

Haie vive, embocage

Score unique

Rang

BRF et broyage des résidus de coton

$0,015 \% \quad 4$

$0,00 \% \quad 5$

$0,018 \%$

Culture annuelle en intercalaire anacardier

$0,016 \% \quad 3$

\begin{tabular}{l|l}
$0,03 \%$ & 1 \\
\hline $0,00 \%$ & 6 \\
\hline
\end{tabular}

